\title{
A rank-one, rigid, simple, prime map
}

\author{
A. DEL JUNCO AND D. J. RUDOLPH \\ Department of Mathematics, University of Toronto, Canada, M5S 1A1; Department \\ of Mathematics, University of Maryland, College Park, Maryland, MA 20742, USA
}

(Received 19 November 1985)

\begin{abstract}
We construct a rank-1 map $T$ similar to Chacon's map [C], [JRS], but with $2^{n+1} n$-blocks in an $(n+1)$-block and a single spacer in the middle. Hence $T$ is rigid and the centralizer of $T, C(T)$, is uncountable. We show $T$ is simple [V], [JR], and hence any factor of $T$ is the algebra of invariant sets of some weakly compact subgroup of $C(T)$. We show $C(T)$ has no such subgroups and hence $T$ is prime. Lastly we show that $T$ is graphic in the sense of [AM].
\end{abstract}

\section{0 . Introduction}

The notion of a 2-fold simple measure-preserving map $T$ of a probability space $(X, \mu)$ was introduced by Veech $[\mathbf{V}]$ under a different name (property S). (See [JR] for a clarification of the history of the terminology). $T$ is 2 -fold simple if any ergodic $T \times T$-invariant measure $\lambda$ on $X \times X$ which has marginals $\mu$ is either $\mu \times \mu$ or a measure $\mu_{S}=(\mathrm{id} \times S) \mu_{\Delta}$ where $S$ is a measure-preserving map commuting with $T$ and $\mu_{\Delta}$ is the diagonal measure over $\mu$. We denote by $C(T)$ the centralizer of $T$, that is, all measure preserving maps commuting with $T$.

Veech showed that if $T$ is simple and $\mathscr{F}$ is a non-trivial $T$-invariant sub- $\sigma$-algebra then there is a weakly compact subgroup $K$ of $C(T)$ such that, up to null sets, $\mathscr{F}=\{A: S A=A \quad \forall S \in C(T)\}$. The main application which he had in mind was that if $C(T)$ has no non-trivial compact subgroups then $T$ is prime - the only invariant $\sigma$-algebras are, up to null sets, the trivial one and the full $\sigma$-algebra.

In [JR] the notion of simplicity was extended to higher orders (and to more general group actions). $T$ is said to be $k$-fold simple if every $T \times \cdots \times T$-invariant ergodic measure on $X^{k}$ is a product of off-diagonal measures, where by an offdiagonal measure on $X^{k^{\prime}}, k^{\prime} \subset\{1, \ldots, k\}$ we mean a measure $\left(\otimes_{i \in k^{\prime}} S_{i}\right) \mu_{\Delta}$ where $S_{i} \in C(T)$ and $\mu_{\Delta}$ is diagonal measure on $X^{k^{\prime}}$. We say $T$ is simple if it is $k$-fold simple of all orders. In [JR] Veech's result together with results of [JR] was applied to obtain new examples of prime maps, for example the time-1 map of any weaklymixing flow with minimal self-joinings (see [JR] for the definition and [JP] for an example of such).

The purpose of this paper is to construct a quite different sort of simple prime map. In fact our example is rigid which means that there is a sequence $\boldsymbol{n}_{\boldsymbol{i}} \rightarrow \infty$ such that $T^{n_{i}} \rightarrow$ id weakly. As is well-known (see [KSS]) this forces the centralizer to be uncountable. As opposed to the time-1 map of a flow our example has a non-locally 
compact centralizer. In the course of establishing 2-fold simplicity we obtain a fairly explicit description of the centralizer.

As shown in $[\mathbf{J R}]$, simplicity has important consequences which go beyond those of mere 2-fold simplicity. We mention just one example; any two simple prime maps are either disjoint or isomorphic. We establish simplicity (of all orders) for our example. In addition the description of the centralizer can be used to show that $T^{m}$ has only the obvious roots (e.g. $T$ has no roots at all) whence $T^{m}$ and $T^{n}$ are non-isomorphic for $|m|>|n|>0$. (It is obvious from the construction that $T$ and $T^{-1}$ are isomorphic). The general theory then implies that $T^{m}$ and $T^{n}$ are disjoint, and much more, in fact.

Finally we consider the topological behaviour of $(X, T)$, which is much simpler. $(X, T)$ is POD in the sense of [FKS] hence it is prime and its topological centralizer is just its powers.

\section{Construction and simplicity of $T$}

The map $T$ will be described as the left shift on a certain closed subset of $\{0,1\}^{z}$ and will be minimal and uniquely ergodic. We begin by listing a sequence of block names, each a finite string of 0's and 1's.

$$
\begin{aligned}
B_{1}= & 010 \\
B_{2}= & B_{1} B_{1} 1 B_{1} B_{1} \\
\vdots & \vdots \\
B_{n+1}= & B_{n}^{2^{n}} 1 B_{n}^{2^{n} .} .
\end{aligned}
$$

As always multiplication of such strings is concatenation. Letting $h_{n}$ be the length of $B_{n}$,

$$
h_{n+1}=2^{n+1} h_{n}+1>2^{n^{2} / 2} .
$$

We represent points in $\{0,1\}^{z}$ by $\bar{x}=x_{-n} \cdots x_{0} \cdots x_{n} \cdots$. We define $X=\{\bar{x}$; for all $i<j, x_{i} x_{i+1} \cdots x_{j}$ occurs as a string of consecutive 0 's and 1's in some $\left.B_{n}\right\}$. This is clearly a closed, shift-invariant subset of $\{0,1\}^{z}$.

For any finite string $a_{0}, a_{1}, \ldots, a_{j-1}$ of 0 's and 1's, we will call $C=\left\{\bar{x} ; x_{k}=a_{k-i}\right.$, $i \leq k \leq j\}$ a cylinder set. The set $(i, i+1, \ldots, j)$ we will call the indices of $C$, and $a_{0}, \ldots, a_{j-1}$ the name of $C$.

A few facts about the $n$-blocks follow easily.

LEMMA 1.1. (i) In any $B_{n}$, two consecutive 1's never occur and three consecutive 0's never occur.

(ii) If $a_{0}, \ldots, a_{l-1}$ is a sequence of consecutive 0 's and 1's in some $B_{n}$, then

$$
\frac{\#\left\{i \in(0, \ldots, l-2) ; a_{i} \neq a_{i+1}\right\}}{l-1} \geq \frac{2}{3}-\frac{2}{l-1} \text {. }
$$

(iii) In $B_{n}^{2}$ the only strings of $h_{n}$ consecutive symbols that are identically $B_{n}$ are the two given copies.

We say a string of 0 's and 1's $a_{0} a_{1} \cdots a_{l-1}$ has a $k$-block cover if there are integers 
$k_{1}, k_{2}<2 h_{k}$ and

$$
a_{k_{1}} \cdots a_{l-k_{2}-1}=\left(\prod_{i=1}^{s-1} B_{k}^{j_{k}} 1\right) B_{k}^{j_{k}},
$$

where all $j_{i} \geq 2$. That is to say except for short $\left(<2 h_{k}\right)$ pieces at either end, $\bar{a}$ can be written as a concatenation of strings of $k$-blocks, each at least a $B_{k}^{2}$, separated by single 1's.

LemMa 1.2. (iv) Any $a_{0} \cdots a_{l-1}$ has at most one $k$-block cover and if it does, then any substring of more than $4 h_{k}$ consecutive symbols also does.

(v) For any $n>k, B_{n}$ can be written uniquely as a concatenation of strings of the form $B_{k}^{2^{k}}$ or $B_{k}^{2^{k+1}}$, separated by single 1 's, the first and last such of the form $B_{k}^{2^{k}}$. Hence any substring of at least $4 h_{k}$ symbols in $B_{n}$ has a unique $k$-block cover.

(vi) For any $\bar{x} \in X, j-i \geq 4 h_{k}, x_{i} \cdots x_{j}$ has a unique $k$-block cover, and hence the entire name $\bar{x}$ has a unique $k$-block cover where the $k$-blocks occur in strings of the form $B_{k}^{2^{k}}$ or $B_{k}^{2^{k+1}}$.

If in this unique $k$-block cover, $x_{0}=1$ corresponds to a 1 between strings of $k$-blocks, then $\bar{x} \in S_{k}$. This defines the set $S_{k}$ as a union of cylinder sets on indices $-2 h_{k}$ to $2 h_{k} . S_{k}$ is open and closed, and $S_{k+1} \subset S_{k}$.

For $\bar{x} \in X$, the occurrences on the $T$ orbit of $\bar{x}$ of points in $S_{k}$ are separated by exactly $h_{k+1}$, or $\left(h_{k+1}+1\right) / 2$ points. Notice $T^{i}(\bar{x}) \in S_{k}$ exactly when index $i$ in the $k$-block cover for $\bar{x}$ lies between two $k$-block strings. Between two consecutive such occurrences, the name of $\bar{x}$ is either $B_{k}^{2^{k}}$ or $B_{k}^{2^{k+1}}$. Thus the set of values $j$ with $T^{j}(\bar{x}) \in S_{k}$ determines $\bar{x} \in X$.

LEMMA 1.3. $(X, T)$ is minimal.

Proof. Any string $a_{0}, \ldots, a_{l-1}$ that is the name of some cylinder $C, C \cap X \neq 0$, occurs in some $B_{k}$. For any $\bar{x} \in X$ there must exist a $-h_{k}-1 \leq j \leq h_{k}$ with $T^{j}(\bar{x}) \in C$.

For any 0,1 -name $a_{0}, \ldots, a_{l-1}$ let $d_{n}(\bar{a})$ be the density in $\cdots B_{n} B_{n} \cdots=B_{n}^{z}$ of occurrences of $\bar{a}$.

LeMmA 1.4. For $\bar{a}=a_{0} \cdots a_{l-1},\left|d_{k+1}(\bar{a})-d_{k}(\bar{a})\right|<2 l / h_{k+1}$.

Proof.

$$
B_{k+1}^{z}=\cdots 1 B_{k}^{2^{k+1}} 1 B_{k}^{2^{k+1}} 1 B_{k}^{2^{k+1}} \cdots .
$$

The difference can only arise within $l$ indices of a 1 .

Corollary 1.5. For any $\bar{a}=a_{0} \cdots a_{l-1}, \lim _{k \rightarrow \infty} d_{k}(\bar{a})=d(\bar{a})$ exists and in fact

$$
\left|d(\bar{a})-d_{k}(\bar{a})\right| \leq \sum_{n=k+1}^{\infty} \frac{2 l}{h_{n}}<\frac{l}{2^{k-1}} .
$$

For a cylinder set $C$, we define $\mu(C)=d(\bar{a}), \bar{a}$ the name of $C$. This finitely additive measure extends to a shift invariant Borel measure on $(X, T, B)$.

Corollary 1.6. $(X, \mu, B, T)$ is uniquely ergodic.

Corollary 1.7. $\mu\left(S_{k}\right)<1 / 2^{k} h_{k}$. 
Proof. Occurrences of $S_{k}$ on the orbit of any $\bar{x} \in X$ are separated by at least $\left(h_{k+1}+1\right) / 2>2{ }^{k} h_{k}$.

Corollary 1.8. For $\bar{x} \in X$;

$$
\lim _{N \rightarrow \infty}\left(\frac{\#\left\{|i| \leq N ; x_{i+h_{k}} \neq x_{i}\right\}}{2 N+1}\right) \leq \frac{1}{2^{k}} .
$$

Proof. If $x_{i+h_{k}} \neq x_{i}$ then for some $j \in\left(i, \ldots, i+h_{k}-1\right), T^{j}(\bar{x}) \in S_{k}$. Hence the above 

$$
\leq \mu\left(\bigcup_{i=0}^{h_{k}-1} T^{-i}\left(S_{k}\right)\right) \leq h_{k} \mu\left(S_{k}\right) \leq \frac{1}{2^{k}} .
$$

That it is actually a limit follows from unique ergodicity.

COROLlaRY 1.9. For any cylinder set $C, \mu\left(T^{h_{k}}(C) \Delta C\right) \stackrel{k}{\rightarrow} 0$, and $T$ is rigid.

COROLLARY 1.10. The weak closure of the powers of $T$ is uncountable and hence $C(T)$ is uncountable [KSS].

In fact $C(T)=$ wkcl $\left(\left\{T^{i}\right\}\right)$ can be concluded on three different levels. First we will see it directly for this example from lemma 1.25. Second, for a strongly rigid rank-1 $T$ such as this the result follows from [CS]. Third, King [K] has shown this holds for any rank-1 map.

Let $n(k, \bar{x})=$ largest $n \leq 0$ with $T^{n}(\bar{x}) \in S_{k}$, the return time under $T^{-1}$ to $S_{k}$. Now define

$$
\begin{aligned}
A_{k} & =\left\{\bar{x} ; x_{n(k, \bar{x})-h_{k+1}} \cdots x_{0} \cdots x_{n(k, \bar{x})+2 h_{k+1}}\right. \\
& =1 B_{k}^{2^{k+1}} 1 B_{k}^{2^{k+1}} 1 B_{k}^{2^{k+1}} 1
\end{aligned}
$$

and

$$
\left.-h_{k}>n(k, \bar{x})>-\left(2^{k+1}-1\right) h_{k}\right\} .
$$

Thus if $\bar{x} \in A_{k}$, in its $k$-block cover index 0 lies in a string of $2^{k+1} B_{k}$ 's, at least a full $B_{k}$ from either end, and the strings of $B_{k}$ 's to either side of this one also are $B_{k}^{2^{k+1}}$.

LEMMA 1.11 .

$$
\mu\left(A_{k}\right)>1-\left(3 h_{k+1}+1\right) \mu\left(S_{k+1}\right)-2 h_{k} \mu\left(S_{k}\right)>1-\frac{1}{2^{k-3}}
$$

Proof. If

$$
-\frac{3 h_{k+1}+1}{2}<n(k+1, \bar{x})<\frac{3 h_{k+1}+1}{2}-h_{k-2}
$$

and

$$
-h_{k}>n(k, \bar{x})>-\left(2^{k}-1\right) h_{k}
$$

then $\bar{x} \in A_{k}$.

Let $E_{k}=\bigcap_{j=k}^{\infty} A_{j}$, those $\bar{x} \in A_{j}$ for all $j \geq k$. Thus $\bar{x} \in E_{k}$ exactly if it is at least $h_{k}$ from a point in $S_{k}$ on its orbit and at least $\left(3 h_{k^{\prime}}+1\right) / 2$ from a point in $S_{k^{\prime}}$ for $k^{\prime}>k$.

Corollary 1.12. (i) $\lim _{k \rightarrow \infty} \mu\left(E_{k}\right)=1$, i.e. for $\mu$-a.e. $\bar{x}$, once $k$ is large enough, $\bar{x} \in E_{k}$.

(ii) For $\mu$-a.e. $\bar{x}, \lim _{k \rightarrow \infty} n(k, \bar{x})=-\infty, \lim _{k \rightarrow \infty} n(k, \bar{x})+h_{k+1}=\infty$. 
Our next step is to show that $T$ is 2 -fold simple, i.e. any $T \times T$ invariant ergodic measure on $X \times X$ is either $\mu \times \mu$ or is supported on the graph of some $S \in C(T)$. To proceed, assume $\lambda$ is such a $T \times T$ invariant ergodic Borel measure on $X \times X$, which must have marginals $\mu$ by corollary 1.6 .

For $\lambda$-a.e. $(\bar{x}, \bar{y})$, once $k$ is large enough both $\bar{x}, \bar{y} \in E_{k}$ and thus among the points

$$
T^{n(k, \bar{x})}(\bar{y}), \ldots, T^{n(k, x)+h_{k+1}-1}(y)
$$

there is exactly one point $T^{n(k, \bar{x})+1}(\bar{y}) \in S_{k}$,

$$
l= \begin{cases}n(k, \bar{y})-n(k, \bar{x}) & \text { if this is } \geq 0 \\ n(k, \bar{y})-n(k, \bar{x})+h_{k+1} & \text { otherwise. }\end{cases}
$$

This point $l$ splits the indices $n(k, \bar{x}), \ldots, n(k, \bar{x})+h_{k+1}-1$ into two pieces

$$
\begin{aligned}
& I=I_{k}(\bar{x}, \bar{y})=\{i ; n(k, \bar{x}) \leq i<l\} \\
& J=J_{k}(\bar{x}, \bar{y})=\left\{i ; l \leq i<n(k, \bar{x})+h_{k+1}\right\} .
\end{aligned}
$$

We define

$$
\begin{aligned}
& U_{k}(\bar{x}, \bar{y})=\bigcup_{i \in I}(T \times T)^{i}(\bar{x}, \bar{y}) \cap\left(E_{k} \times X\right) \cap\left(X \times E_{k}\right) \\
& V_{k}(\bar{x}, \bar{y})=\bigcup_{i \in J}(T \times T)^{i}(\bar{x}, \bar{y}) \cap\left(E_{k} \times X\right) \cap\left(X \times E_{k}\right),
\end{aligned}
$$

i.e. the corresponding pieces of orbit as long as both coordinates are in $E_{k}$. Notice that if $\left(\bar{x}^{\prime}, \bar{y}^{\prime}\right) \in U_{k}(\bar{x}, \bar{y}) \cup V_{k}(\bar{x}, \bar{y})$ then

$$
U_{k}(\bar{x}, \bar{y})=U_{k}\left(\bar{x}^{\prime}, \bar{y}^{\prime}\right)
$$

and

$$
V_{k}(\bar{x}, \bar{y})=V_{k}\left(\bar{x}^{\prime}, \bar{y}^{\prime}\right),
$$

and both of these are strings of consecutive points on orbits.

Define

$$
W_{k}=\left\{(\bar{x}, \bar{y}) ;(\bar{x}, \bar{y}) \text { is in the smaller of } U_{k}(\bar{x}, \bar{y}), V_{k}(\bar{x}, \bar{y})\right\},
$$

(one must in fact be smaller).

LEMMA 1.13. $\varlimsup W_{k}$ is $\lambda$-a.s. $T \times T$ invariant, hence

$$
\lambda\left(\overline{\lim } W_{k}\right)=0 \text { or } 1 .
$$

Proof. Split $W_{k}$ into two sets

$$
\begin{aligned}
& W_{k}^{\prime}=\left\{(\bar{x}, \bar{y}) \in W_{k} ; \text { the block } U_{k}(\bar{x}, \bar{y}),\right. \\
& \left.\quad \text { or } V_{k}(\bar{x}, \bar{y}) \text { containing }(\bar{x}, \bar{y}) \text { is shorter than } 2 h_{k}\right\} \\
& W_{k}^{\prime \prime}=W_{k} \backslash W_{k}^{\prime} .
\end{aligned}
$$

Now if $(\bar{x}, \bar{y}) \in W_{k}^{\prime}$, then one of $\bar{x}, \bar{y}$ is in the set

$$
\bigcup_{i=-3 h_{k}}^{3 h_{k}} T^{-1}\left(S_{k}\right)
$$

a set of measure $<6 / 2^{k-1}$ so $\lambda\left(\overline{\lim } W_{k}^{\prime}\right)=0$. 
Points $(\bar{x}, \bar{y}) \in W_{k}^{\prime \prime}$ come in segments on a $T \times T$ orbit at least $h_{k}$ long, hence the same is true of $\bigcup_{j=k}^{\infty} W_{j}^{\prime \prime}$. Thus

$$
\lambda\left(\bigcup_{j=k}^{\infty} W_{j}^{\prime \prime}\right) \cap(T \times T)\left(\bigcup_{j=k}^{\infty} W_{j}^{\prime \prime}\right) \geq\left(1-\frac{2}{h_{k}}\right) \lambda\left(\bigcup_{j=k}^{\infty} W_{j}^{\prime \prime}\right),
$$

and

$$
\lambda\left(\widetilde{\lim } W_{j}^{\prime \prime} \cap(T \times T)\left(\overline{\lim } W_{j}^{\prime \prime}\right)\right)=\left(\overline{\lim } W_{j}^{\prime \prime}\right)
$$

and $\varlimsup^{\prime \prime m} W_{j}^{\prime \prime}$ is $\lambda$-a.s. $T \times T$ invariant.

That $T$ is 2 -fold simple is a result of the following dichotomy.

THEOREM 1. (a) If $\lambda\left(\overline{\lim } W_{j}\right)=1$ then $\lambda=\mu \times \mu$.

(b) If $\lambda\left(\overline{\lim } W_{j}\right)=0$ then $\lambda$ is supported on the graph of some $S \in$ weak closure $\left\{T^{i}\right\}$.

We prove this in steps. The basic tool to prove (a), and furthermore that $C(T)$ contains no compact subgroups, and the simplicity of all orders for $T$, is the construction of a certain sequence of maps.

$$
\hat{\phi}_{k}: \hat{D}_{k} \rightarrow \hat{R}_{k}, \quad \hat{D}_{k}, \hat{R}_{k} \subset X \times X,
$$

so that for any particular $(\bar{x}, \bar{y}) \in \hat{D}_{k}$, there is a $t>k$ with $(\bar{x}, \bar{y}) \in U_{t}(\bar{x}, \bar{y})$ and

$$
\hat{\phi}_{k}(\bar{x}, \bar{y})=(T \times T)^{j h_{k}}(\bar{x}, \bar{y}) \in V_{t}(\bar{x}, \bar{y}) .
$$

Notice that for such a $\hat{\phi}_{k}$, and $(\bar{x}, \bar{y}) \in \hat{D}_{k}$, if $C, D$ are cylinder sets whose indices lie in $\left(-h_{k}+1, \ldots, h_{k}-1\right)$ then $(\bar{x}, \bar{y}) \in C \times D$ iff

$$
\hat{\phi}_{k}(\bar{x}, \bar{y}) \in C \times T^{-1}(D) \text {. }
$$

This leads to the following fact.

LEMMA 1.14. Suppose there exist $\lambda$-preserving 1-1 Borel maps $\hat{\phi}_{k}: \hat{D}_{k} \rightarrow \hat{R}_{k}$ so that for $(\bar{x}, \bar{y}) \in \hat{D}_{k}$ there is a $t>k$ with $(\bar{x}, \bar{y}) \in U_{t}(\bar{x}, \bar{y})$ and

$$
\hat{\phi}_{k}(\bar{x}, \bar{y})=(T \times T)^{j h_{k}}(\bar{x}, \bar{y}) \in V_{t}(\bar{x}, \bar{y}) .
$$

Furthermore suppose

$$
\lim \lambda\left(\hat{D}_{k} \cup \hat{R}_{k}\right)>0 .
$$

It follows that $\lambda$ is $\mathrm{id} \times T^{-1}$ invariant and hence is $\mu \times \mu$.

Proof. If $C, D$ are cylinder sets whose indices lie in $\left(-\left(h_{k}-1\right) / 2, \ldots,\left(h_{k}-1\right) / 2\right)$ and $N_{k}=\left(h_{k}-1\right) / 2$, then

$$
\frac{1}{N_{k}} \sum_{i=0}^{h_{k}-1} \chi_{C \times D}\left((T \times T)^{i}(\bar{x}, \bar{y})\right)=\frac{1}{N_{k}} \sum_{i=0}^{h_{k}-1} \chi_{C \times T^{-1}(D)}\left((T \times T)^{i} \hat{\phi}_{k}(\bar{x}, \bar{y})\right) .
$$

As $\hat{\phi}_{k}$ is $\lambda$-preserving and $1-1$, and $\lim \lambda\left(\hat{D}_{k} \cup \hat{R}_{k}\right)>0$, fixing $C, D$, we can select points $\left(\bar{x}_{k}, \bar{y}_{k}\right) \in \hat{D}_{k}$ so that

$$
\begin{gathered}
\frac{1}{N_{k}} \sum_{i=0}^{h_{k}-1} \chi_{C \times D}\left((T \times T)^{i}\left(\bar{x}_{k}, \bar{y}_{k}\right)\right) \stackrel{k}{\rightarrow} \lambda(C \times D) \\
\frac{1}{N_{k}} \sum_{i=0}^{h_{k}-1} \chi_{C \times T^{-1}(D)}\left((T \times T)^{i} \hat{\phi}_{k}\left(\bar{x}_{k}, \bar{y}_{k}\right)\right) \stackrel{k}{\rightarrow} \lambda\left(C \times T^{-1}(D)\right)
\end{gathered}
$$

by the Birkhoff ergodic theorem. Hence $\lambda$ is id $\times T^{-1}$-invariant on all cylinder sets, 
hence all Borel sets. Thus $\lambda$ is $T^{i} \times T^{j}$-invariant for all $i, j$. But as $T$ is uniquely ergodic, so is this $Z^{2}$ action on $X \times X$ with unique invariant measure $\mu \times \mu$.

Under the assumption $\lambda\left(\widetilde{\lim } W_{k}\right)=1$ we proceed to construct the $\hat{\phi}_{k}$. First we define maps $\phi_{k}$ as follows.

If $(\bar{x}, \bar{y}) \in U_{k}(\bar{x}, \bar{y})$, let,

where

$$
\phi_{k}(\bar{x}, \bar{y})=(T \times T)^{j h_{k}}(\bar{x}, \bar{y})
$$

$$
j=\left[\frac{\min \left(\# I_{k}(\bar{x}, \bar{y}), \# J_{k}(\bar{x}, \bar{y})\right)}{h_{k}}\right]+1,
$$

if this puts $\phi_{k}(\bar{x}, \bar{y})$ in $V_{k}(\bar{x}, \bar{y})$. (The square brackets here denote the greatest integer function.)

The value $j$ above is determined solely by where the cut between the indices in $I$ and $J$ occurs. Furthermore, if $(\bar{x}, \bar{y}) \in W_{k}$, then either $(\bar{x}, \bar{y}) \in \operatorname{Dom}\left(\phi_{k}\right)=D_{k}$ or $(\bar{x}, \bar{y}) \in$ Range $\left(\phi_{k}\right)=R_{k}$ or $\bar{y}$ is within $3 h_{k}$ of an occurrence of $S_{k}$ on its $T$ orbit.

LEMMA 1.15. (i) The maps $\phi_{k}$ are $\lambda$-preserving and 1-1.

(ii) $D_{k} \cap R_{k}=\varnothing$.

(iii) If $(\bar{x}, \bar{y}) \in D_{k} \cap D_{k^{\prime}}, k^{\prime}>k$ then $\phi_{k}(\bar{x}, \bar{y}) \in D_{k^{\prime}}$.

(iv) If $(\bar{x}, \bar{y}) \in R_{k} \cap R_{k^{\prime}}, k^{\prime}>k$, then $\phi_{k}^{-1}(\bar{x}, \bar{y}) \in R_{k^{\prime}}$.

(v) $W_{k} \subset D_{k} \cup R_{k} \cup X \times \sum_{i=-3 h_{k}}^{3 h_{k}} T^{i}\left(S_{k}\right)$

Proof. That $\phi_{k}$ is $1-1, \lambda$-preserving and $D_{k} \cap R_{k}=\varnothing$ are easily seen.

To obtain (iii), suppose $(\bar{x}, \bar{y}) \in D_{k} \cap D_{k^{\prime}}$. We know then that $\phi_{k}(\bar{x}, \bar{y})=\left(\bar{x}^{\prime}, \bar{y}^{\prime}\right)$ has $\bar{x}^{\prime}, \bar{y}^{\prime} \in E_{k} \subset E_{k^{\prime}-1}$ and hence that $\left(\bar{x}^{\prime}, \bar{y}^{\prime}\right) \in U_{k^{\prime}}(\bar{x}, \bar{y})$.

Now $\left(\bar{x}^{\prime}, \bar{y}^{\prime}\right) \in D_{k^{\prime}}$ as long as $\left(\bar{x}^{\prime \prime}, \bar{y}^{\prime \prime}\right)=\phi_{k^{\prime}}\left(\bar{x}^{\prime}, \bar{y}^{\prime}\right) \in V_{k^{\prime}}(\bar{x}, \bar{y})$. We know $\left(\bar{x}^{\prime \prime}, \bar{y}^{\prime \prime}\right)$ is in the block given by indices $J$, what we must check is that $\bar{x}^{\prime \prime}, \bar{y}^{\prime \prime} \in E_{k^{\prime}}$.

We get $\bar{x}^{\prime \prime} \in E_{k^{\prime}}$, as it is still a full $B_{k^{\prime}}$ from the next occurrences of $S_{k^{\prime}}$ on the orbit of $\bar{x}$, lying as it does between the same occurrences of $S_{k^{\prime}}$ on the orbit as $\bar{x}$. The $k$-block containing $\bar{y}^{\prime}$ is not the last in a string in the $k^{\prime}$-block cover of $\bar{y}$, as $\bar{y}^{\prime} \in E_{k^{\prime}-1}$. Hence the same is true of $\bar{y}^{\prime \prime}$ and $\bar{y}^{\prime \prime} \in E_{k^{\prime}}$. (iv) is analogous to (iii) and (v) we have already seen.

We use the maps $\phi_{k}$ to construct $\hat{\phi}_{k}$ as follows. Select $K$ so large that

$$
\lambda\left(\bigcup_{j=k+1}^{K}\left(D_{j} \cup R_{j}\right)\right)>\lambda\left(\overline{\lim }\left(D_{j} \cup R_{j}\right)\right)-1 / k
$$

For $(\bar{x}, \bar{y}) \in \bigcup_{j=k+1}^{K} D_{j} \cup R_{j}$, select the largest $k<j \leq K$ with $(\bar{x}, \bar{y}) \in D_{j} \cup R_{j}$. If $(\bar{x}, \bar{y}) \in D_{j}$ then define

$$
\hat{\phi}_{k}(\bar{x}, \bar{y})=\phi_{j}(\bar{x}, \bar{y}) \text {. }
$$

Let $\hat{D}_{k}$ be the domain of definition of $\hat{\phi}_{k}$, and $\hat{R}_{k}$ its range.

LemMA 1.16. $\hat{D}_{k} \cup \hat{R}_{k}=\bigcup_{j=k+1}^{K} D_{j} \cup R_{j}$ and $\hat{\phi}_{k}$ is $1-1, \lambda$-preserving.

Proof. If $j$ is the largest value with $(\bar{x}, \bar{y}) \in D_{j} \cup R_{j}$ and $(\bar{x}, \bar{y}) \in R_{j}$, then $j$ is the largest value with $\phi_{j}^{-1}(\bar{x}, \bar{y}) \in D_{j}$ by (iv) of lemma 1.15 . Thus

$$
\hat{D}_{k} \cup \hat{R}_{k}=\bigcup_{j=k+1}^{K} D_{j} \cup R_{j} \text {. }
$$


If $\hat{\phi}_{k}(\bar{x}, \bar{y})=\phi_{j}(\bar{x}, \bar{y})=\phi_{j^{\prime}}\left(\bar{x}^{\prime}, \bar{y}^{\prime}\right)=\hat{\phi}_{k}\left(\bar{x}^{\prime}, \bar{y}^{\prime}\right)$ where $j^{\prime}>j$ then by (iv) of lemma 1.5 , $(\bar{x}, \bar{y}) \in D_{j^{\prime}}$ conflicting with the choice of $j . \hat{\phi}_{k}$ is $\lambda$-preserving as it is equal to $\phi_{j}$ on disjoint pieces of $\hat{D}_{k}$.

Corollary 1.17 ((a) of theorem 1). If $\lambda\left(\overline{\lim } W_{k}\right)=1$, then $\lambda=\mu \times \mu$.

Proof. $\lambda\left(\hat{D}_{k} \cup \hat{R}_{k}\right)>\lambda\left(\overline{\lim } D_{j} \cup R_{j}\right)-1 / k$ so $\lim \lambda\left(\hat{D}_{k} \cup \hat{R}_{k}\right) \geq \lambda\left(\overline{\lim } D_{j} \cup R_{j}\right) \geq$ $\lambda\left(\overline{\lim } W_{k}\right)=1$ and the result follows from lemma 1.14.

We can now assume $\lambda\left(\overline{\lim } W_{k}\right)=0$, i.e. for $\lambda$-a.e. $\bar{x}, \bar{y}$, once $k$ is sufficiently large, $(\bar{x}, \bar{y})$ belongs to the larger of $U_{k}(\bar{x}, \bar{y})$ and $V_{k}(\bar{x}, \bar{y})$. Set

$$
G_{k}=\left(\bigcup_{j=k}^{\infty} W_{k}^{c}\right) \cap\left(E_{k} \times X\right) \cap\left(X \times E_{k}\right),
$$

and we now can assume $\lim _{k \rightarrow \infty} \lambda\left(G_{k}\right)=1$. Define

$$
f_{k}(\bar{x}, \bar{y})=n(k, \bar{x})-n(k, \bar{y}),
$$

the shift of the two string $1 B_{k}^{2^{k+1}}$ containing $\bar{x}$ and $\bar{y}$ relative to one another, when both are in such $\left(f_{k}\right.$ though is defined on all $\left.\boldsymbol{X} \times \boldsymbol{X}\right)$.

The function $f_{k}$ maps the measure $\lambda$ to a measure on $\left(-h_{k+1}, \ldots, h_{k+1}\right)$. Our investigation from here out will consist of understanding what these measures $f_{k}^{*}(\lambda)$ look like.

LeMmA 1.18. If $(\bar{x}, \bar{y}),\left(\bar{x}^{\prime}, \bar{y}^{\prime}\right) \in G_{k}$ and $f_{k+1}(\bar{x}, \bar{y})=f_{k+1}\left(\bar{x}^{\prime}, \bar{y}^{\prime}\right)$ then

$$
f_{k}(\bar{x}, \bar{y})=f_{k}\left(\bar{x}^{\prime}, \bar{y}^{\prime}\right) \text {. }
$$

Proof. As $f_{k+1}(\bar{x}, \bar{y})=f_{k+1}\left(\bar{x}^{\prime}, \bar{y}^{\prime}\right)$, the blocks $1 B_{k+1}^{2^{k+2}} 1$ containing them are shifted the same amounts relative to one another. Hence

$$
\begin{aligned}
& \# I_{k}(\bar{x}, \bar{y})=f_{k+1}(\bar{x}, \bar{y}) \bmod \left(h_{k+1}\right), \\
& \# J_{k}(\bar{x}, \bar{y})=h_{k+1}-\# I_{k}(\bar{x}, \bar{y}),
\end{aligned}
$$

and as $(x, y) \in G_{k}$, we know

$$
f_{k}(\bar{x}, \bar{y})=\left\{\begin{aligned}
\# I_{k}(\bar{x}, \bar{y}) & \text { if }(\bar{x}, \bar{y}) \in U_{k}(\bar{x}, \bar{y}) \\
-\# J_{k}(\bar{x}, \bar{y}) & \text { if }(\bar{x}, \bar{y}) \in V_{k}(\bar{x}, \bar{y}) .
\end{aligned}\right.
$$

Note: the larger of $I, J$ is the same as the larger of $U, V$.

Corollary 1.19. If $(\bar{x}, \bar{y}),\left(\bar{x}^{\prime}, \bar{y}^{\prime}\right) \in G_{k}$ and for some $k^{\prime}>k, f_{k^{\prime}}(\bar{x}, \bar{y})=f_{k^{\prime}}\left(\bar{x}^{\prime}, \bar{y}^{\prime}\right)$ then $f_{k}(\bar{x}, \bar{y})=f_{k}\left(\bar{x}^{\prime}, \bar{y}^{\prime}\right)$.

Corollary 1.20. $f_{k}$ is constant $\lambda$-a.e. on $G_{k}$.

Proof. If $(\bar{x}, \bar{y})$ and $\left(\bar{x}^{\prime}, \bar{y}^{\prime}\right)$ are both on the same $T \times T$ orbit and in some $G_{k}$, then once $k^{\prime}$ is sufficiently large,

$$
T^{n\left(k^{\prime}, \bar{x}\right)}(\bar{x})=T^{n\left(k^{\prime}, \bar{x}^{\prime}\right)}\left(\bar{x}^{\prime}\right) \quad \text { and } \quad T^{n\left(k^{\prime}, \bar{y}\right)}(\bar{y})=T^{n\left(k^{\prime}, \bar{y}^{\prime}\right)}\left(\bar{y}^{\prime}\right) .
$$

Thus

$$
f_{k^{\prime}}(\bar{x}, \bar{y})=f_{k^{\prime}}\left(\bar{x}^{\prime}, \bar{y}^{\prime}\right)
$$

and $f_{k}$ is invariant under the induced map $(T \times T)_{G_{k}}$ which is ergodic for $\lambda / G_{k}$. 
We will now, w.l.o.g, assume $f_{k}$ is strictly constant on $G_{k}, f_{k}\left(G_{k}\right)=n_{k}$. Thus we have an increasing sequence of sets $G_{k}$ with $f_{k} ; G_{k} \rightarrow n_{k}$. Recalling the measures $f_{k}^{*}(\lambda)$ on $\left(-h_{k+1}, \ldots, h_{k+1}\right)$, this says they become ever more concentrated on a single point. More precisely if we define

$$
f ; X \times X \rightarrow \prod_{k=1}^{\infty}\left(-h_{k+1}, \ldots, h_{k+1}\right)=Y
$$

by $F(\bar{x}, \bar{y})=\left(f_{1}(\bar{x}, \bar{y}), f_{2}(\bar{x}, \bar{y}), \ldots\right)$ then $\lambda$ projects to a measure $f^{*}(\lambda)$ on $Y$. For any infinite sequence $n_{1}, n_{2}, \ldots$, set

$$
S_{k}\left(n_{1}, n_{2} \cdots\right)=\left\{\bar{y} \in Y ; y_{i}=n_{i}, i \geq k\right\} .
$$

We call a Borel measure $\lambda$ on $Y$ an asymptotic point mass on $n_{1}, n_{2} \cdots$ if

$$
\lim _{k \rightarrow \infty} \lambda\left(S_{k}\left(n_{1}, n_{2} \cdots\right)\right)=1 \text {. }
$$

COROLlary 1.21. If $\lambda\left(\overline{\lim } W_{k}\right)=0$ then $f^{*}(\lambda)$ is an asymptotic point mass on the sequence $f_{1}\left(G_{1}\right), f_{2}\left(G_{2}\right) \cdots$.

Denote this sequence by $n_{k}=f_{k}\left(G_{k}\right)$.

COROLlARY 1.22. $\lim _{k \rightarrow \infty} n_{k} / h_{k+1}=0$.

Proof. For $(\bar{x}, \bar{y}) \in G_{k}$, first suppose $n_{k}>0$. Thus $(\bar{x}, \bar{y}) \in U_{k}(\bar{x}, \bar{y})$ and $f_{k}\left(V_{k}(\bar{x}, \bar{y})\right)=$ $h_{k+1}-n_{k}$. Hence

$$
\begin{aligned}
\frac{f_{k}^{*}(\lambda)\left(n_{k}^{c}\right)}{f_{k}^{*}(\lambda)\left(n_{k}\right)} \geq \frac{\lambda\left(\left\{(T \times T)^{j}(\bar{x}, \bar{y}) ;(\bar{x}, \bar{y}) \in G_{k}, i \in J_{k}(\bar{x}, \bar{y})\right\}\right)}{\lambda\left(\left\{(T \times T)^{i}(\bar{x}, \bar{y}) ;(\bar{x}, \bar{y}) \in G_{k}, i \in I_{k}(\bar{x}, \bar{y})\right\}\right)} \\
=\frac{\# J_{k}(\bar{x}, \bar{y})}{\# I_{k}(\bar{x}, \bar{y})}=\frac{f_{k}\left(U_{k}(\bar{x}, \bar{y})\right)}{f_{k}\left(V_{k}(\bar{x}, \bar{y})\right)}=\frac{n_{k}}{h_{k+1}-n_{k}} \geq 0 .
\end{aligned}
$$

If $n_{k}<0,(\bar{x}, \bar{y}) \in V_{k}(\bar{x}, \bar{y})$ and similar reasoning gives

$$
\frac{f_{k}^{*}(\lambda)\left(n_{k}^{c}\right)}{f_{k}^{*}(\lambda)\left(n_{k}\right)}=\frac{-n_{k}}{h_{k+1}+n_{k}} \geq 0 \quad\left(n_{k}^{c}=\text { complement of }\left\{n_{k}\right\}\right) .
$$

As $\left(f_{k}^{*}(\lambda)\left(n_{k}^{c}\right)\right) /\left(f_{k}^{*}(\lambda)\left(n_{k}\right)\right) \stackrel{k}{\rightarrow} 0$ the result follows.

Given that $f^{*}(\lambda)$ is an asymptotic point mass, we now attempt to define a Borel map $S ; X \rightarrow X$ on whose graph $\lambda$ is supported.

For $\bar{x} \in X$ define a cylinder set $N_{k}(\bar{x})$ whose indices are

$$
\left(\max \left(n(k, \bar{x}), n(k, \bar{x})-n_{k}\right), \ldots, \min \left(n(k, \bar{x})+h_{k+1}, n(k, \bar{x})-n_{k}+h_{k+1}\right)\right)
$$

and whose name is the 0,1 -name of $T^{-n_{k}}(\bar{x})$ across these indices.

If it is the case that for some $k_{0}$ and all $k \geq k_{0}$, the indices of $N_{k+1}(\bar{x})$ contain those of $N_{k}(\bar{x})$ and increase in $k$ to all of $\mathbb{Z}$, and furthermore

$$
N_{k+1}(\bar{x}) \subset N_{k}(\bar{x})
$$

i.e. the names agree where they overlap, then $\bigcap_{k} N_{k}(\bar{x})=S(\bar{x})$ is a single point in $X$.

It is an easy fact that the set of $\bar{x} \in X$ where $S$ is defined is a $T$-invariant Borel set, $S$ is a Borel map and $T S(\bar{x})=S T(\bar{x})$. 
Furthermore we can define a Borel map $\hat{S}$ similarly but by translating by $n_{k}$, instead of $-n_{k}$.

It follows that $\bar{y}=S(\bar{x})$ iff $\bar{x}=\hat{S}(\bar{y})$, and so where defined $S$ is $1-1$ and $\hat{S}=S^{-1}$.

LEMMA 1.23. If for some $k_{0},(\bar{x}, \bar{y}) \in G_{k_{0}}$ then $\bar{y}=S(\bar{x}), \bar{x}=S^{-1}(\bar{y})$.

Proof. As $(\bar{x}, \bar{y}) \in G_{k_{0}}$, for all $k \geq k_{0}$

$$
f_{k}(\bar{x}, \bar{y})=n(k, \bar{x})-n(k, \bar{y})=n_{k} .
$$

Thus the name of $N_{k}(\bar{x})$ is

$$
y_{\max (n(k, x), n(k, y))} \cdots y_{\min \left(n(k, x)+h_{k+1}, n(k, y)+h_{k+1}\right)} .
$$

These blocks in the name of $\bar{y}$ increase to all of $\bar{y}$.

Corollary 1.24. $S$ and $S^{-1}$ are defined $\mu$-a.e. and $\lambda(\operatorname{graph}(S))=1$.

LEMMA 1.25. For any cylinder set $C$,

$$
\lim _{k \rightarrow \infty} \mu\left(S^{-1}(C) \Delta T^{n_{k}}(C)\right)=0 .
$$

Proof. Suppose the indices of $C$ are in $(-N, N)$. Given $\varepsilon>0$, once $k$ is large enough,

$$
\begin{aligned}
& \lambda\left(\left\{(\bar{x}, S(\bar{x})) \in G_{k} ; \max (n(k, \bar{x}), n(k, S(\bar{x}))<-N,\right.\right. \\
& \left.\quad \min \left(n(k, \bar{x})+h_{k+1}, n\left(k, S(\bar{x})+h_{k+1}\right)>N\right\}\right)>1-\varepsilon .
\end{aligned}
$$

For such an $x, T^{-n_{k}}(x) \in C$ iff $S(x) \in C$. Thus

$$
\mu\left(S^{-1}(C) \Delta T^{n_{k}}(C)\right)<\varepsilon .
$$

Corollary 1.26. ((b) of theorem 1). $S$ is $\mu$-preserving and in the weak closure of $\left\{T^{i}\right\}$, and for any Borel set $A, X \times X$

$$
\lambda(A)=\int_{x} \chi_{A}(\bar{x}, S(\bar{x})) d \mu .
$$

Proof. The first two remarks follow from lemma 1.25. Letting $\lambda=\int_{x} \lambda_{x} d_{\mu}(x)$ be the disintegration of $\lambda$ over its first coordinate, as $\lambda(\operatorname{graph}(S))=1, \lambda_{x}(S(\bar{x}))=1$ for $\mu$-a.e. $x$. Thus

$$
\lambda(A)=\int_{x} \lambda_{x}(A)_{k} d \mu=\int_{x} \chi_{A}(\bar{x}, S(\bar{x})) d \mu .
$$

Notice that for free we obtain that $C(T)=$ weak closure $\left\{T^{i}\right\}$ as certainly any $S \in C(T)$ gives rise to a $\lambda_{S}$, supported on graph $(S)$, and as such a $\lambda_{S}$ is unique to $S$, puts $S \in$ weak closure $\left\{T^{i}\right\}$. This, of course, is King's theorem [K] for our particular example.

\section{Primality of $T$}

Veech's theorem, which we mentioned in the introduction, now gives the following corollary to the 2-fold simplicity of $T$.

COROLlary 2.1. If $G \subset B$ is a T-invariant factor algebra, then there is a weakly compact subgroup $H \subseteq(C T)$ so that $A \in G$ iff $\mu(S(A) \Delta A)=0$ for all $S \in H$.

Proof. See [V]. 
Our next task is to show that $C(T)$ has no non-trivial compact subgroups, and hence $T$ is prime.

To begin this discussion, consider the set $\Lambda$ of $T \times T$ invariant Borel probability measures on $X \times X$. This is a convex, weakly compact subspace of the vector space of all Borel measures, and has as its extreme points the ergodic joinings. The projection $f^{*}$ to the probability measures on $Y$ is a homeomorphism in the weak topology to its range, (that $f^{*}(\lambda)$ determines $\lambda$ is an easy exercise). We can identify those $\lambda \in \Lambda$ which are supported on the graph of some $S \in C(T)$.

LEMMA 2.2. $\lambda \in \Lambda$ is supported on the graph of some $S \in C(T)$ iff $f^{*}(\lambda)$ is an asymptotic point mass.

Proof. If $\lambda$ is so supported, the result is just theorem 1 and corollary 1.21. Suppose $\lambda$ is an asymptotic point mass on $\left\{n_{1}, n_{2} \cdots\right\}$. Now $\lambda$ has an ergodic decomposition

$$
\lambda=\alpha(\mu \times \mu)+(1-\alpha) \int_{C(T)} \lambda_{S} d \tau(S),
$$

where $\lambda_{S}$ is supported on graph $(S)$ and $\tau$ is a Borel probability measure on $C(T)$.

$$
f^{*}(\lambda)=\alpha f^{*}(\mu \times \mu)+(1-\alpha) \int_{C(T)} f^{*}\left(\lambda_{s}\right) d \tau(s) .
$$

As $f^{*}(\lambda)$ is an asymptotic point mass on some $\left\{n_{i}\right\}, \alpha=0$ and for $\tau$-a.e. $S, T^{n_{i}} \rightarrow S$ weakly. Thus $\lambda$ is a point mass on some $S \in C(T)$ and $\lambda=\lambda_{S}$.

To show that $C(T)$ has no compact subgroups all we need do is show that for any $S \neq$ id in $C(T)$, weak closure $\left(\left\{f^{*}\left(\lambda_{S^{i}}\right)\right\}\right)$ contains measures which are not asymptotic point masses. As this weak closure is compact, all we need is to find a sequence of powers $t_{i}$ so that $f^{*}\left(\lambda_{S^{t_{i}}}\right)$ cannot have an asymptotic point mass as an accumulation point. This argument uses the same approach seen in lemmas 1.15, 1.16, building maps $\phi_{k}$ and then $\hat{\phi}_{k}$.

LEMMA 2.3. For $S \in C(T)$ and $k>0$ suppose there is a $\mu$-preserving 1-1 Borel map $\hat{\phi}_{k} ; \hat{D}_{k} \rightarrow \hat{R}_{k}$, both Borel subsets of $X$, so that for any $\bar{x} \in \hat{D}_{k}$ there is a $t>k$ with $(\bar{x}, S(\bar{x})) \in U_{t}\left(\bar{x}, S(\bar{x})\right.$ and $\hat{\phi}_{k}(\bar{x})=T^{j h_{1}}(\bar{x})$ has $\left(\hat{\phi}_{k}(\bar{x}), S\left(\hat{\phi}_{k}(\bar{x})\right)\right) \in V_{t}(\bar{x}, S(\bar{x}))$. It follows that for all $j<k$, if $\bar{x}, S(\bar{x}) \in E_{j}$ then

$$
f_{j}\left(\hat{\phi}_{k}(\bar{x}), S\left(\hat{\phi}_{k}(\bar{x})\right)\right)=f_{j}(x, S(\bar{x}))-1 \text {. }
$$

Proof. If $\bar{x}, S(\bar{x}) \in E_{j}$ then $n(j, \bar{x}), n(j, S(\bar{x}))>1$ and

$$
\begin{aligned}
n\left(j, S\left(\hat{\phi}_{k}(\bar{x})\right)\right. & =n(j, \bar{x}), \\
n\left(j, S\left(\hat{\phi}_{k}(\bar{x})\right)\right) & =n(j, S(\bar{x}))+1 .
\end{aligned}
$$

Corollary 2.4. Suppose that for some sequence $S_{k} \in C(T)$ there is, for each $S_{k}, a$ $\mu$-preserving 1-1 Borel map $\hat{\phi}_{k} ; \hat{D}_{k} \rightarrow \hat{R}_{k}$ as described in lemma 2.3. Furthermore suppose $\lim \mu\left(\hat{D}_{k}\right)=\alpha>0$. The $S_{k}$ then can have no accumulation points in $C(T)$.

Proof. For any $j$, if $k>j$ and $\bar{x} \in \hat{D}_{k}$,

$$
f_{j}\left(\hat{\phi}_{k}(\bar{x}), S_{k}\left(\hat{\phi}_{k}(\bar{x})\right)\right)=f_{j}\left(\bar{x}, S_{k}(\bar{x})\right)-1,
$$


the largest mass $f_{j}^{*}\left(\lambda_{S_{k}}\right)$ can give to any single point is

$$
\left.1-\left(\frac{\mu\left(\hat{D}_{k} \cup \hat{R}_{k}\right)}{2}\right)-\mu\left(E_{j}^{c}\right)\right) .
$$

Thus for any accumulation point $\lambda$ of the $\lambda_{S_{k}}$, the largest mass $f_{j}^{*}\left(\lambda_{S_{k}}\right)$ can give to any single point is

$$
1-\frac{\mu\left(\hat{D}_{k} \cup \hat{R}_{k}\right)}{2}-\mu\left(E_{j}^{c}\right) .
$$

Thus for any accumulation point $\lambda$ of the $\lambda_{S_{k}}$, the largest mass $f_{j}^{*}(\lambda)$ can give to any point is $1-\left((\alpha / 2)-\mu\left(E_{j}^{c}\right)\right)$. As $\mu\left(E_{j}^{c}\right) \stackrel{j}{\rightarrow} 0, \lambda$ cannot be an asymptotic point mass.

Now all that remains is to find a sequence $t_{k}$ so that $S_{k}=S^{t_{k}}$ satisfies this corollary.

Fix $S \neq$ id. Thus $f^{*}\left(\lambda_{S}\right)$ is an asymptotic point mass on $\left\{n_{1}, n_{2}, \ldots\right\}$, w.l.o.g. $n_{i} \neq 0$ and $\lim _{i \rightarrow \infty} n_{i} / h_{i+1}=0$ by corollary 1.22 . Fix the value $k$ and select $k^{\prime}>k$ so that:

(a) $1 / 20>n_{k^{\prime}} / h_{k^{\prime}+1}>n_{k^{\prime \prime}} / h_{k^{\prime \prime}+1}$ for all $k^{\prime \prime}>k^{\prime}$;

(b) $f^{*}\left(\lambda_{s}\right)\left(\left\{\left\{j_{i}\right\} \in Y ; j_{i}=n_{i}, i \geq k^{\prime}\right\}\right)>0.99$; and

(c) $\mu\left(E_{k^{\prime}}^{c}\right)<0.01$, and $\mu\left(S_{k^{\prime}}\right)<0.01 / h_{k}$.

Select the integer $t_{k}$ so that

$$
\frac{1}{4}<\frac{\left|t_{k} n_{k^{\prime}}\right|}{h_{k^{\prime}+1}}<\frac{1}{3} .
$$

Define

$$
\begin{aligned}
0_{k}=\left\{\bar{x} ; \bar{x}, S^{t_{k}}(\bar{x}) \in E_{k^{\prime}}, f_{j}(\bar{x}, S(\bar{x}))=n_{j}, j \geq k^{\prime}\right. \text { and } \\
0 \notin\left(\operatorname { m a x } \left(n\left(k^{\prime}, \bar{x}\right), n\left(k^{\prime}, \bar{x}\right)+t_{k} n_{k^{\prime}}, \ldots,\right.\right. \\
\\
\left.\left.\min \left(n\left(k^{\prime}, \bar{x}\right)+h_{k^{\prime}+1}, n\left(k^{\prime}, \bar{x}\right)+h_{k^{\prime}+1}+t_{k} n_{k^{\prime}}\right)\right)\right\} .
\end{aligned}
$$

Thus, if $n_{k^{\prime}}>0$, then $\bar{x} \in 0_{k}$ if it is within $t_{k} n_{k^{\prime}}$ of the right end of its block $1 B_{k^{\prime}}^{2^{k^{\prime}+1}} 1$, and if $n_{k^{\prime}}<0$, it is within this amount of the left end. As $\left|t_{k} n_{k^{\prime}}\right|>h_{k^{\prime}+1} / 4, \mu\left(0_{k}\right)>0.22$.

We will define $\hat{\phi}_{k}$ so that

$$
\hat{D}_{k} \cup \hat{R}_{k} \supseteq 0_{k} .
$$

For a.e. $\bar{x} \in 0_{k}$, once $j>k^{\prime}$ is large enough, we will have all of $\bar{x}, S(\bar{x}), \ldots, S^{i}{ }^{\prime}(\bar{x}) \in E_{j}$ and $f_{j}\left(S^{i}(\bar{x}), S^{i+1}(\bar{x})\right)=n_{j}$ for $i=0, \ldots, t_{k}-1$. Let $j_{0}+1$ be the smallest such $j \geq k^{\prime}$. Figure 1 illustrates how the $t_{k}$ different blocks $1 B_{j_{0}+1}^{2^{j^{+}+2}} 1$ look.

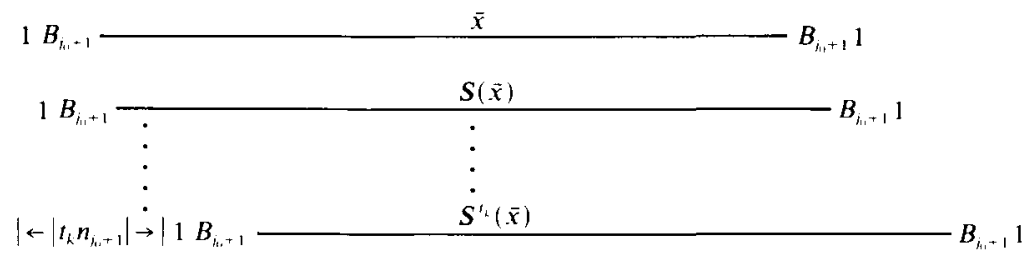

FIGURE 1

As $\bar{x}, \ldots, S^{t^{\prime}}(\bar{x}) \in E_{j_{0}+1}$, all have 0,1 -name on indices

$$
\left(n\left(j_{0}, S^{i}(\bar{x})\right) \cdots n\left(j_{0}, S^{i}(\bar{x})+h_{j_{0}+1}\right)\right.
$$


equal to $1 B_{j_{0}}^{2_{0}{ }^{+1}} 1$. The 0,1 -name for $S^{i+1}(\bar{x})$ has its long periodic mid section in figure 1 shifted relative to that in $S^{i}(\bar{x})$ by $n_{j_{0}+1} \bmod \left(h_{j_{0}+1}\right)$. As $f_{j_{0}}(\bar{x}, S(\bar{x}))=n_{j_{0}}$,

$$
n_{j_{0}+1} \bmod \left(h_{j_{0}+1}\right)= \begin{cases}n_{j_{0}} & \text { if } n_{j_{0}}>0 \\ n_{j_{0}}+h_{j_{0}+1} & \text { otherwise. }\end{cases}
$$

Thus the ' 1 ' indicating the occurrence of $S_{j_{0}}$ in the 0,1 -name of $S^{i}(\bar{x})$ on indices $\left(n\left(j_{0}, \bar{x}\right), \ldots, n\left(j_{0}, \bar{x}\right)+h_{j_{0}+1}\right)$ is successively shifted by $n_{j_{0}}$ as $i$ increases.

If these 1's, as $i$ increases, do not cross from one side of $\bar{x}$ to the other, then first, $j_{0}>k^{\prime}$, as this is not true of $k^{\prime}$, and second as both $\bar{x}, S^{t_{k}}(\bar{x}) \in E_{j_{0}}$, so are all $S^{i}(\bar{x})$ and $f_{j_{0}}\left(S^{i}(\bar{x}), S^{i+1}(\bar{x})\right)=n_{j_{0}}$ for $i=0, \ldots, t_{k}-1$, contradicting the minimality of $j_{0}$. Thus

$0 \notin\left(\max \left(n\left(j_{0}, \bar{x}\right), n\left(j_{0}, \bar{x}\right)+t_{k} n_{j_{0}}\right), \ldots, \min \left(n\left(j_{0}, \bar{x}\right)+h_{j_{0}+1}, n\left(j_{0}, \bar{x}\right)+h_{j_{0}+1}+t_{k} n_{j_{0}}\right)\right.$, and as

$$
\frac{\left|t_{k} n_{j_{0}}\right|}{h_{j_{0}+1}}<\frac{\left|t_{k} n_{k^{\prime}}\right|}{j_{k^{\prime}+1}}<\frac{1}{3},
$$

$\left(\bar{x}, S^{t_{k}}(\bar{x})\right)$ belongs to the smaller of $U_{j_{0}}\left(\bar{x}, S^{t_{k}}(\bar{x})\right), V_{j_{0}}\left(\bar{x}, S^{t_{k}}(\bar{x})\right),\left(\right.$ both $\bar{x}, S^{t_{k}}(\bar{x})$ are in $E_{j_{0}}$ ).

As defined, $U_{j_{0}}\left(\bar{x}, S^{t_{k}}(\bar{x})\right)$ and $V_{j_{0}}\left(\bar{x}, S^{t_{k}}(\bar{x})\right)$ are subsets of $X \times X$, but as they sit on graph $(S)$, their projection onto the first coordinate is $1-1$, so just as preceeding lemma 1.15 , we can define a map $\phi_{j_{0}}$ taking as much of $U_{j_{0}}\left(\bar{x}, S^{t_{k}}(\bar{x})\right)$ to $V_{j_{0}}\left(\bar{x}, S^{t_{k}}(\bar{x})\right)$ as possible by a shift by a multiple of $h_{j_{0}}$. These maps satisfy all of lemma 1.15 , except $(v)$ is replaced by

$$
0_{k} \subset \bigcup_{j_{0}=k^{\prime}}^{\infty}\left(D_{j_{0}} \cup R_{j_{0}} \cup \bigcup_{i=-3 h_{j_{0}}}^{3 h_{j_{0}}} T^{i}\left(S^{-t_{k}}\left(S_{j_{0}}\right)\right)\right) .
$$

Thus

$$
\mu\left(\bigcup_{j_{0}=k^{\prime}}^{\infty} D_{j_{0}} \cup R_{j_{0}}\right)>0.15
$$

Construct $\hat{\phi}_{k}$ exactly as preceeding lemma 1.16 except truncate so that

$$
\mu\left(\bigcup_{j_{0}=k^{\prime}}^{K} D_{j_{0}} \cup R_{j_{0}}\right)>0.1 .
$$

The conclusions of lemma 1.16 still hold for $\hat{\phi}_{k}$, i.e. $\hat{\phi}_{k}$ is $1-1, \mu$-preserving and $\lim \mu\left(D_{k}\right) \geq 0.1$.

LEMMA 2.5. Given any $S \neq$ id in $C(T)$ there exists a sequence $t_{k}$ so that $S^{t_{k}}$ can have no weak accumulation points in $C(T)$.

Proof. We have demonstrated the hypotheses of corollary 2.4 .

THEOREM 2. $T$ is prime.

Corollary 2.6. $T$ is weakly mixing.

Proof. It is, of course, a joke to use this much machinery to prove so obvious a fact. We have not assumed it, of course, never actually claiming $\mu \times \mu$ was an ergodic measure for $T \times T$. 
The proof is, of course, that any non-weakly mixing map has non-trivial factor algebras, unless it is a finite rotation of prime order which $T$ is not.

\section{Higher order simplicity and some consequences}

Our next step is to show that $T$ is simple of all orders. Knowing $T$ is 2-fold simple, all we need show is that any $m$-fold ergodic joining $\lambda$ on $X^{m}$ that is pairwise independent on coordinate algebras must be $\mu^{m}$ (in the vocabulary of [JR],T is pairwise independently determined). If we can show that any such joining is id $^{m-1} \times T^{-1}$ invariant we will be done, as permuting coordinates implies any such $\lambda$ is $T^{j_{1}} \times T^{j_{2}} \times \cdots \times T^{j_{m}}$ invariant. This $\mathbb{Z}^{m}$ action on $X^{m}$ is uniquely ergodic as $T$ is. The proof that such a pairwise independent $\lambda$ is $\mathrm{id}^{m-1} \times T^{-1}$-invariant again follows the format of lemma 1.14 , by constructing maps $\hat{\phi}_{k}$.

Suppose $\lambda$ is an ergodic $m$-fold joining on $X^{m}$. For $w=\left(\bar{x}^{1}, \bar{x}^{2}, \ldots, \bar{x}^{m}\right) \in X^{m}$, with all $\bar{x}^{i} \in E_{k}$, the interval of indices $n\left(k, \bar{x}^{1}\right), \ldots, n\left(k, \bar{x}^{1}\right)+h_{K+1}-1$ contains indices $l_{2}, l_{3}, \ldots, l_{m-1}, l_{m}$ with $T^{l_{i}}\left(\bar{x}^{i}\right) \in S_{k}$.

Letting $l_{0}=n\left(k, \bar{x}^{1}\right)$ and $l_{i}=n\left(k, \bar{x}^{1}\right)+h_{k+1}$, the index $l_{m}$ will lie between two nearest such

$$
l_{a} \leq l_{m}<l_{b}, \quad a, b \in\{0, \ldots, m-1\}
$$

LEMMA 3.1.

$$
\begin{gathered}
\lambda\left(\left\{w ; \bar{x}^{i} \in E_{k}, l_{a} \leq 0<l_{b}, l_{m}-l_{a}>\frac{h_{k+1}}{10 m}, l_{b}-l_{m}>\frac{h_{k+1}}{10 m}\right\}\right) \\
>\left(\frac{4}{100 m}-\frac{1}{2^{k-1}}\right) \mu\left(E_{k}\right)-(m-1) \mu\left(E_{k}^{c}\right)
\end{gathered}
$$

Proof. If all $\bar{x}^{i} \in E_{k}, \bar{x}^{m}$ is within a fraction $1 / 10 m$ of the end of its block $1 B_{k}^{2^{k+1}} 1$, but for all $i \neq m, \bar{x}^{i}$ is further than a fraction $2 / 10 m$ from the ends of its block $1 B_{k,}^{2^{k+1}} 1$, then $w$ is in the set in question.

By pairwise independence, for $i \neq m, \lambda\left(\left\{w ; \bar{x}^{m}, \bar{x}^{i} \in E_{k}, \bar{x}^{m}\right.\right.$ is within a fraction $1 / 10 m$ of the end of its block and $\bar{x}^{i}$ is within a fraction $2 / 10 m$ of the end of its block\}) is less than or equal to:

$$
\frac{\left(\frac{2 h_{k+1}}{10 m}-3 h_{k}-1\right)}{h_{k+1}} \frac{\left(\frac{4 h_{k+1}}{10 m}-3 h_{k}-1\right)}{h_{k+1}} \mu\left(E_{k}\right)^{2} \leq\left(\frac{4}{10 m}-\frac{1}{2^{k-1}}\right)^{2} \mu\left(E_{k}\right)^{2} .
$$

Thus $\lambda$ (the set in question) is greater than or equal to:

$$
\begin{aligned}
& \left(\frac{2}{10 m}-\frac{1}{2^{k-1}}\right) \mu\left(E_{k}\right)-(m-1)\left(\mu\left(E_{k}^{c}\right)\right)-(m-1)\left(\frac{4}{10 m}-\frac{1}{2^{k-1}}\right)^{2} \mu\left(E_{k}\right)^{2} \\
& \geq\left(\frac{4}{100 m}-\frac{1}{2^{k-1}}\right) \mu\left(E_{k}\right)-(m-1) \mu\left(E_{k}^{c}\right) .
\end{aligned}
$$

Setting $0_{k}=\left\{w ; \bar{x}^{i} \in E_{k}, l_{a} \leq 0<l_{b}, l_{m}-l_{a}>h_{k+1} / 10 m, l_{b}-l_{m}>h_{k+1} / 10 m\right\}$, we conclude $\lim \lambda\left(0_{k}\right) \geq 0.04 / m$. 
Letting $T \times T \times \cdots \times T=\hat{T}$, for $w \in 0_{k}$, the index $l_{m}$ splits the indices $l_{a}, l_{a}+1$, $\ldots, l_{b}-1$ into two sets

$$
\begin{aligned}
& I_{k}(w)=\left\{i ; l_{a} \leq i<l_{m}\right\} \\
& J_{k}(w)=\left\{i ; l_{m} \leq i<l_{b}\right\} .
\end{aligned}
$$

Except for those $i \in I_{k}(w) \cup J_{k}(w)$ within $\left(3 h_{k}+1\right) / 2$ of $l_{a}, l_{m}$ or $l_{b}$, all $\hat{T}^{i}(w)$ are also in $0_{k}$.

Let $U_{k}(w)=\left\{T^{i}(w) \in 0_{k} ; i \in I_{k}(w)\right\}$ and $V_{k}(w)=\left\{T^{i}(w) \in 0_{k} ; i \in J_{k}(w)\right\}$. Let $W_{k}=$ $\left\{w \in 0_{k} ; w\right.$ is in the smaller of $\left.U_{k}(w), V_{k}(w)\right\}$.

LEMMA 3.2. $\varlimsup W_{k}$ is $\lambda$-a.s. $\hat{T}$-invariant and as

$\varlimsup W_{k}=1$.

$$
\lambda\left(W_{k}\right) \geq \lambda\left(0_{k}\right) \cdot\left(\left(\left(h_{k+1} / 10 m\right)-3 h_{k}-1\right) / h_{k+1}\right),
$$

Proof. The proof that $W_{k}$ is $\lambda$-a.s. $\hat{T}$-invariant is analogous to lemma 1.13 , only now we have $m$ coordinates instead of just two. As $\min \left(\# U_{k}(w), \# V_{k}(w)\right) \geq$ $\left(h_{k+1} / 10 m\right)-3 h_{k}-1$, and $\# I_{k}(w)+\# J_{k}(w)=h_{k+1}$,

$$
\begin{aligned}
\lambda\left(W_{k}\right) & \geq \lambda\left(0_{k}\right)\left(\frac{h_{k+1}}{10 m}-3 h_{k}-1\right) / h_{k+1} \\
& >\lambda\left(0_{k}\right)\left(\frac{1}{10 m}-\frac{1}{2^{k-1}}\right) .
\end{aligned}
$$

Thus $\overline{\lim } \lambda\left(W_{k}\right)>(1 / 10 m) \lim \lambda\left(0_{k}\right)>0$.

As in our previous two constructions, we now define maps $\phi_{k} ; D_{k} \rightarrow R_{k}$ mapping as much of $U_{k}(w)$ to $V_{k}(w)$ as possible by a shift by a multiple of $h_{k}$. The conclusions of lemma 1.15 will again hold, except that (v) is replaced by

$$
W_{k} \subset D_{k} \cup R_{k} \cup\left\{w \text {; some } \bar{x}^{i} \text {, is within } 3 h_{k} \text { of } S_{k} \text { on its } T \text { orbit }\right\} \text {. }
$$

This is still enough to imply

$$
\lambda\left(\varlimsup \overline{\lim } D_{k} \cup R_{k}\right)=1 .
$$

As before lemma 1.16, we construct $\hat{\phi}_{k} ; \hat{D}_{k} \rightarrow \hat{R}_{k}$ from the $\phi_{k}$, and the conclusions of lemma 1.16 still hold. An $m$-fold version of lemma 1.14 now leads to the following conclusion.

LEMMA 3.3. If $\lambda$ is an ergodic $m$-fold joining of $T$ with itself, whose coordinate algebras are pairwise independent, then $\lambda$ is $\mathrm{id}^{m-1} \times T^{-1}$-invariant.

THEOREM 3. $T$ is simple of all orders.

Applying theorem 4.1 of [JR] we now know that any joining of $T$ to an arbitrary $S$ arises as the relatively independent joining of the $k$-fold product of $T$ and $S$ over the factor algebra $T^{k \odot}$ of symmetric sets, which $S$ must have as a factor. Furthermore, by corollary 4.6 of [JR] if we were to construct two 'different' versions of $T$, both simple and prime, then they must either be disjoint or isomorphic. If by 'different' all we meant was that the position of the single spacer among the $2^{k+1} k$-blocks in a $(k+1)$-block varied from one construction to the other, then in fact the maps would be isomorphic. We leave it to the reader's cleverness to see why. All our arguments really only depended on $h_{k+1} / h_{k}$ growing fast enough. We leave open 
the question whether choosing sufficiently different numbers of $k$-blocks in a $(k+1)$-block would lead to non-isomorphic maps.

We conclude this section by showing how the results at hand can be used to show that for $|m|>|n|>0, T^{m}$ and $T^{n}$ are disjoint. By theorem 6.1 of [JR] both $T^{m}$ and $T^{n}$ are simple and $C\left(T^{m}\right)=C\left(T^{n}\right)=C(T)$. By corollary 4.6 of [JR], then $T^{m}$ and $T^{n}$ are either disjoint or isomorphic. We rule out the possibility of isomorphism by showing that $T^{n}$ has no $m$ th root. Indeed if $S^{m}=T^{n}$ then $S \in C\left(T^{n}\right)=C(T)$. Then, in the notation of $\S 2, f^{*}\left(\lambda_{s}\right)$ is an asymptotic point mass on a sequence $\left\{k_{i}\right\}$. The fact that $k_{i} / h_{i+1} \rightarrow 0$ makes it clear that $f^{*}\left(\lambda_{s^{m}}\right)$ is an asymptotic point mass on $\left\{m k_{i}\right\}$. But $f^{*}\left(\lambda r^{n}\right)$ is evidently an asymptotic point mass on the constant sequence $\{n\}$ so we have $n=m k_{i}$ for large $i$ which is impossible since $|m|>|n|$. This establishes the disjointness of $T^{m}$ and $T^{n}$.

Now $T$ and $T^{-1}$ are evidently isomorphic via an involution $\phi$. If $\psi$ is any other isomorphism then $\psi^{-1} \phi \in C(T)$ so $\psi=\phi S$ for some $S \in C(T)$. Note that $\phi S \phi^{-1}=S^{-1}$ since $S$ is a weak limit of powers of $T$, so $\phi S \phi S=\mathrm{id}$, that is $\psi$ is also an involution. We now have the following description of invariant measures for $\otimes_{i \in k} T^{n_{i}}$ on $X^{k}$, $n_{i} \neq 0, k$ a finite set.

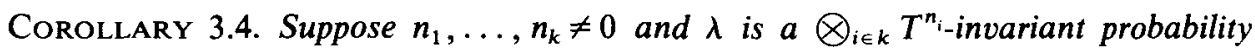
measure on $X^{k}$. Then $\lambda$ is a product of measures of the form $\otimes_{i \in k} S_{i} \mu_{\Delta}$ on $X^{k^{\prime}}, k^{\prime} \subset k$, where $\mu_{\Delta}$ is diagonal measure on $X^{k}$ and each $S_{i}$ is either in $C(T)$ or of the form $\phi S_{i}^{\prime}$, $S_{i}^{\prime} \in C(T)$. Moreover $n_{i}$ is constant on $k^{\prime}$ and $S_{i} \in C(T)$ either precisely for those ifor which $n_{i}>0$ or precisely for those $i$ for which $n_{i}<0$.

Proof. This follows from the fact that all $T^{m}, m \neq 0$, are simple and prime, together with proposition 5.3 of [ JR] and the above remarks. (See also the proof of proposition 6.7 of $[\mathbf{J R}]$ and the remarks following that proof.)

\section{Topological behaviour}

In this section we will show that $(X, T)$ is POD in the sense of [FKS]. In particular the set of continuous maps commuting with $T$ is just $\left\{T^{i}\right\}$, so although there are non-trivial weakly convergent sequences of powers of $T$ there are no non-trivial uniformly convergent sequences. Another consequence of the POD property is that $(X, T)$ is topologically prime.

We start by distinguishing two special points in $X, \bar{x}^{0}$ whose 0,1 -name on indices $-h_{k}-1$ to $h_{k}$ is $B_{k} B_{k}$, and $\bar{x}^{1}$ whose name on indices $-h_{k}-1$ to $h_{k}+1$ is $B_{k} 1 B_{k}$. Notice these two points have identical names on negative indices, but have names differing by a shift by 1 on positive indices.

LEMMA 4.1. If $\bar{x}$ and $\bar{y}$ do not lie one in the orbit of $\bar{x}^{\circ}$ and the other in the orbit of $\bar{x}^{1}$ and $n(k, \bar{x})=n(k, \bar{y})$ for all $k$ then $x_{i}=y_{i}$ for all $i$, i.e. $\bar{x}=\bar{y}$.

Proof. Now $n(k, \bar{x})=n(k, \bar{y})$ for all $k$, implies $x_{i}=y_{i}$ if

$$
n(k, \bar{x}) \leq i \leq n(k, \bar{x})+\frac{h_{k+1}-1}{2} \text {. }
$$

If $n(k, \bar{x}) \stackrel{k}{\rightarrow}-\infty$ and $n(k, \bar{x})+\left(h_{k+1}-1\right) / 2 \stackrel{k}{\rightarrow} \infty$ we are done. As $n(k, \bar{x})$ is non- 
increasing and $n(k, \bar{x})+\left(h_{k+1}-1\right) / 2$ is non-decreasing,

(a) if $\lim _{k \rightarrow \infty} n(k, \bar{x})=n>-\infty$, then $T^{n}(\bar{x})=T^{n}(\bar{y})=\bar{x}^{1}$; and

(b) if $\lim _{k \rightarrow \infty} n(k, \bar{x})+\left(h_{k+1}-1\right) / 2=n<\infty$, then $T^{n+1}(\bar{x})=\bar{x}^{1}$ or $\bar{x}^{0}$ and $T^{n+1}(\bar{y})=$ $\bar{x}^{1}$ or $\bar{x}^{0}$. As both must be on the same orbit of $\bar{x}^{1}$ and $\bar{x}^{0}, \bar{x}=\bar{y}$.

For $\bar{x} \in X$, let $j_{i}(k, \bar{x})$ be the values $j<0$ in decreasing order in $i$ with $T^{j}(\bar{x}) \in S_{k}$.

LEMMA 4.2. If $\bar{x}$ and $\bar{y}$ do not one lie on the orbit of $\bar{x}^{0}$ and the other $\bar{x}^{1}$, and for some $k>0, j_{0} \in Z, j_{i}(k, \bar{x})-j_{i}(k, \bar{y})=j_{0}$ for all $i$, then $\bar{y}=T^{-j_{0}}(\bar{x})$.

Proof. The values $j_{i}(k, \bar{x})$ determine the values $n\left(k^{\prime}, \bar{x}\right)$ for $k^{\prime} \geq k$, and hence $k^{\prime}<k$. For $k^{\prime} \geq k, n\left(k^{\prime}, \bar{y}\right)=n\left(k^{\prime}, \bar{x}\right)-j_{0}$ so

$$
n\left(k^{\prime}, T^{j_{0}}(\bar{y})\right)=n\left(k^{\prime}, \bar{x}\right)
$$

But then $n\left(k^{\prime}, T^{j_{0}}(\bar{y})\right)-n\left(k^{\prime}, \bar{x}\right)$ for all $k^{\prime}$ and $T^{j_{0}}(\bar{y})=\bar{x}$.

Fix a pair of points $(\bar{x}, \bar{y}) \in X \times X$, on distinct orbits, at most one of which is on the orbit of $\bar{x}^{0}$ or $\bar{x}^{1}$. If it is the case that one is on the orbit of $\bar{x}^{0}$ or $\bar{x}^{1}$, be sure it is a right shift of $\bar{x}^{0}$ or $\bar{x}^{1}$ by multiplying $(\bar{x}, \bar{y})$ by a sufficiently high negative power of $T \times T$, and using this pair instead of $(\bar{x}, \bar{y})$.

We define a collection of pairs of cylinder sets $I(\bar{x}, \bar{y})$ where $\{C, D\} \in I(\bar{x}, \bar{y})$ if for some $j \leq 0,\left(T^{j}(\bar{x}), T^{j}(\bar{y})\right) \in C \times D$ and the indices of both $C$ and $D$ are contained in $(-\infty,-j)$, i.e. the name of $C \times D$ appears in the negative indices of $(\bar{x}, \bar{y})$.

From the restriction placed on $(\bar{x}, \bar{y})$, any finite set of negative indices lies completely inside the indices of $k$-blocks containing 0 in the $k$-block covers of $\bar{x}$ and $\bar{y}$, if $k$ is large enough.

Thus for $\{C, D\} \in I(\bar{x}, \bar{y})$, once $k$ is large enough, the indices of $T^{-j}(C)$ and $T^{-j}(D)$ lie inside the intersection of the indices of the $k$-blocks containing 0 of $\bar{x}$ and $\bar{y}$ (see figure 2).

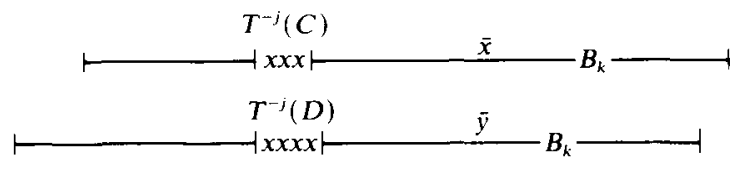

0

FIGURE 2

In these two $k$-block covers, move one block at a time to the left. If as we so proceed, every time the $\bar{x}$ name crosses a 1 in between two $B_{k}$ 's, the $\bar{y}$ name also does, then $j_{i}(k, \bar{x})=j_{i}(k, \bar{y})+j_{0}$ for all $i$, and by lemma $3.2, x=T^{j_{0}}(\bar{y})$, contrary to choice.

We conclude that finally one of the two names crosses an occurrence of $S_{k}$ and the other does not. Focus on the first such occurrence (see figure 3 ).

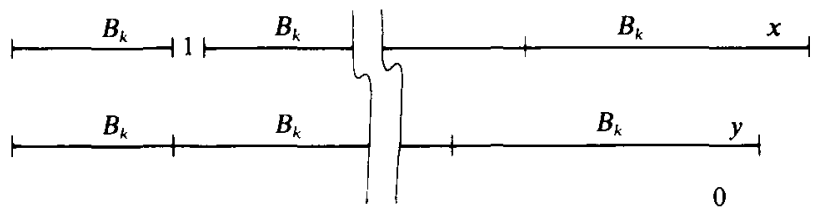

FIGURE 3 
In each $k$-block in the name of $\bar{x}$ we see the name of $C$, and in $\bar{y}$, that of $D$. In these next two $k$-blocks, the relative positions though have shifted by one place. Thus one of the two pairs $\{C, T(D)\}$ or $\left\{C, T^{-1}(D)\right\}$ is in $I(\bar{x}, \bar{y})$.

LeMmA 4.3. If $\{C, D\} \in I(\bar{x}, \bar{y})$ then one of $\{C, T(D)\}$ or $\left\{C, T^{-1}(D)\right\} \in I(\bar{x}, \bar{y})$.

LEMMA 4.4. If $J \subset \mathbb{Z}$ is finite and $C, D$ are such that for all $i \in J,\left\{C, T^{i}(D)\right\} \in I(\bar{x}, \bar{y})$ then either

(a) for all $i \in J,\left\{C, T^{i-1}(D)\right\} \in I(\bar{x}, \bar{y})$; or

(b) for all $i \in J,\left\{C, T^{i+1}(D)\right\} \in I(\bar{x}, \bar{y})$.

Proof. For each $i \in J$ there is a $j(i) \leq 0$ so that

$$
(x, y) \in \bigcap_{i \in J} T^{-j(i)}(C) \times T^{-j(i)+i}(D) .
$$

Select $N<0$ so that all the indices of all the $T^{-j(i)}(C)$ and $T^{-j(i)+i}(D)$ are contained in $(N, N+1, \ldots, 0)$. Let $C^{\prime}$ be the cylinder set with name $x_{n} x_{n+1} \cdots x_{0}$ on indices $n$ to 0 and similarly $D^{\prime}$ have name $y_{n} \cdots y_{0}$ on indices $n$ to 0 . Now $\left\{C^{\prime}, D^{\prime}\right\} \in I(\bar{x}, \bar{y})$ and $C^{\prime} \times D^{\prime} \subset \bigcap_{i \in J} T^{-j(i)}(C) \times T^{-j(i)+i}(D)$. By lemma 4.3 either:

(a) $\left\{C^{\prime}, T^{-1}(D)\right\} \in I(\bar{x}, \bar{y})$, and so for some $j<0$

$$
(\bar{x}, \bar{y}) \in T^{-j}\left(C^{\prime}\right) \times T^{-j-1}\left(D^{\prime}\right) \subset \bigcap_{i \in J} T^{-j(i)-j}(C) \times T^{-j(i)-j+i-1}(D)
$$

and for $i \in J,\left\{C, T^{i-1}(D)\right\} \in I(\bar{x}, \bar{y})$, or:

(b) $\left\{C^{\prime}, T\left(D^{\prime}\right)\right\} \in I(\bar{x}, \bar{y})$ in which case for all $i \in J$,

$$
\left\{C, T^{i+1}(D)\right\} \in I(\bar{x}, \bar{y}) \text {. }
$$

Corollary 4.5. For any $\{C, D\} \in I(\bar{x}, \bar{y})$, either

(a) $\mathbb{Z}^{+} \subset\left\{i ;\left\{C, T^{i}(D)\right\} \in I(\bar{x}, \bar{y})\right\}$; or

(b) $\mathbb{Z}^{-} \subset\left\{i ;\left\{C, T^{i}(D)\right\} \in I(\bar{x}, \bar{y})\right\}$

(or perhaps both).

Proof. By lemma 4.4, $\left\{i ;\left\{C, T^{i}(D)\right\} \in I(\bar{x}, \bar{y})\right\}$ cannot exclude both a positive and negative value unless it is $\varnothing$. It cannot be empty though as $T$ is minimal.

THEOREM 4. If $\bar{x}, \bar{y} \in X$ are on distinct orbits and at most one is on the orbit of $\bar{x}^{1}$ or $\bar{x}^{0}$ the $T \times T$ orbit closure of $(\bar{x}, \bar{y})$ is all of $X \times X$.

Proof. Translate $(\bar{x}, \bar{y})$ if necessary so that $I(\bar{x}, \bar{y})$ is defined. Fix cylinder sets $U, V$. As $T$ is uniquely ergodic, on any orbit there is a maximum distance $N$ between occurrences of $U$ or $V$. Assume $N$ is larger than the number of indices of either $U$ or $V$.

Let

$$
C=x_{-5 n} x_{-5 n+1} \cdots x_{0}
$$

and

$$
D=y_{-5 n} y_{-5 n+1} \cdots y_{0}
$$

so $\{C, D\} \in I(\bar{x}, \bar{y})$.

There must be values

$$
-4 n<j(1) \leq-3 n<j(2) \leq-2 n<j(3)<-n
$$


with $T^{-j(1)}(\bar{x}) \in U, T^{-j(2)}(\bar{y}) \in V, T^{-j(3)}(\bar{x}) \in U$. Thus

$$
C \times D \subset\left(T^{j(1)}(U) \times T^{j(2)}(V)\right) \cap\left(T^{j(3)}(U) \times T^{j(2)}(V)\right) .
$$

Either $\left\{C, T^{j(1)-j(2)}(D)\right\}$ or $\left\{C, T^{j(3)-j(2)}(D)\right\} \in I(\bar{x}, \bar{y})$ by corollary 4.5 . In either case $\{U, V\} \in I(\bar{x}, \bar{y})$ and the closure of just the past orbit of $(\bar{x}, \bar{y})$ is $X \times X$.

COROllary 4.6. $(X, T)$ is POD in the sense of [FKS]. In particular $(X, T)$ is graphic in the sense of $[\mathbf{A M}]$.

Proof. We already know that $(X, T)$ is minimal and measure theoretically, hence also topologically, weakly mixing. Thus we only have that for any $\bar{x}, \bar{y} \in X$ on different orbits the orbit closure of $(x, y)$ contains the graph of $T^{m}$ for some $m \neq 0$. If at most one of $\bar{x}$ or $\bar{y}$ is $\bar{x}^{0}$ or $\bar{x}^{1}$, theorem 4 gives much more. If one is $\bar{x}^{0}$ and the other is $\bar{x}^{1}$ then the orbit closure contains the graphs of $T^{m}$ and $T^{m+1}$ for some $m$.

More remains to be done concerning $(X, T)$. For example, can the results of [JK] on Chacon's map be proved here and can $(X, T)$ be shown to have topological minimal self-joinings in the stronger sense of $[\mathbf{J}]$.

\section{REFERENCES}

[AM] J. Auslander \& N. Markley. Graphic flows and multiple disjointness. Trans. Amer. Math. Soc. To appear.

[C] R. V. Chacón. Weakly mixing transformations which are not strongly mixing. Proc. Amer. Math. Soc. 22 (1969), 559-562.

[CS] R. V. Chacón \& T. Schwartzbaur. Commuting point transformations. Z. Wahrsch. verw. Geb. 11 (1969), 277-287.

[FKS] H. Furstenberg, H. Keynes \& L. Shapiro. Prime flows in topological dynamics. Israel J. Math. 14 (1973), 26-38.

[J] A. del Junco. A definition of minimal self-joinings in topological dynamics. To appear.

[JK] A. del Junco \& M. Keane. On generic points in the Cartesian square of Chacón's transformation. Ergod. Th. \& Dynam. Sys., 5 (1985), 59-69.

[JP] A. del Junco \& K. Park. An example of a measure-preserving flow with minimal self-joining. J. d'Analyse Math. 42 (1983), 199-211.

[JR] A. del Junco \& D. J. Rudolph. On ergodic actions whose self-joinings are graphs. Ergod. Th. \& Dynam. Sys. To appear.

[JRS] A. del Junco, A. M. Rahe \& L. Swanson. Chacón's automorphism has minimal self-joinings. J. d'Analyse Math. 37, (1980), 276-284.

[KSS] A. B. Katok, Ya. G. Sinai \& A. M. Stepin. Theory of dynamical systems and general transformation groups with invariant measures J. Soviet Math. (1977), 974-1065. Translated from Itogi Nauki Teh. Ser. Mat. Anal. 13 (1975), 129-262.

[K] J. King. The commutant is the weak closure of the powers, for rank-1 transformations. Ergod. Th. \& Dynam. Sys. To appear.

[V] W. A. Veech. A criterion for a process to be prime. Monatsh. Math. 94 (1982), 335-341. 